\title{
Search-match tool for atomic pair distribution functions and Crystallography Open Database
}

\author{
P. Juhás ${ }^{1}$ and L. Pouchard ${ }^{1}$ \\ ${ }^{1}$ Computational Science Initiative, Brookhaven National Laboratory, Upton, New York 11973, USA \\ Search-match programs are routinely used for powder diffraction data to identify phases, verify \\ sample purity and/or find the best initial structure for a detailed structure refinement. We present \\ a prototype search-match tool that brings similar capabilities for atomic pair distribution function \\ (PDF) analysis. We have utilized the Crystallography Open Database (COD) ${ }^{1}$ and the DiffPy-CMI \\ software $^{2}$ to compute theoretical x-ray PDFs for the entire COD collection and to extract their char- \\ acteristic features such as the nearest neighbor distance, positions of the strongest peak, and their \\ probability moments at selected intervals. These characteristics were entered into Elasticsearch ${ }^{3}$ \\ system together with selected fields from the COD entries allowing to do search-match queries for \\ experimental PDF curves. The large set of simulated PDFs enabled further analytics such as group- \\ ing of near-equivalent COD entries, and assessment of scaling-independent features in the PDFs to \\ facilitate similarity searches even for phases that are not yet in COD. Elasticsearch is a powerful \\ and extensible search engine which allows to search and collate multiple document collections using \\ either programmatic or natural-language queries. We will demonstrate such cross analytics with \\ experimental records from the XPD powder diffraction beamline at the NSLS-II synchrotron and \\ also discuss real-time PDF analysis during data acquisition at XPD.
}

${ }^{1}$ S. Gražulis, D. Chateigner, R. T. Downs, A. F. T. Yokochi, M. Quirós, L. Lutterotti, E. Manakova, J. Butkus, P. Moeck, and A. Le Bail, J. Appl. Crystallogr. 42 (2009), 10.1107/S0021889809016690.

2 P. Juhás, C. L. Farrow, X. Yang, K. R. Knox, and S. J. L. Billinge, Acta Crystallogr. A 71, 562 (2015).

3 "Elasticsearch - Open Source Search \& Analytics," https://elastic.co (2018). 